\title{
MobiLE: Um Ambiente Multiagente de Aprendizagem Móvel Baseado em Algoritmo Genético para Apoiar a Aprendizagem Ubiqua
}

\author{
Title: MobiLE: A Mobile Learning Multi Agent Environment Based in Genetic Algo- \\ rithm to Support Ubiquitous Learning
}

Luiz Cláudio Nogueira da Silva Superintendência de Tecnologia da Informação e Comunicação (SUTIC) Universidade Federal Rural do Semiárido (UFERSA)

luizclaudio@ufersa.edu.br

\author{
Francisco Milton Mendes Neto \\ Programa de Pós-Graduação em Ci- \\ ência da Computação (PPgCC) \\ Universidade Federal Rural do Semi- \\ árido (UFERSA) \\ miltonmendes@ufersa.edu.br
}

\author{
Luiz Jácome Júnior \\ Programa de Pós-Graduação em Ci- \\ ência da Computação (PPgCC) \\ Universidade Federal Rural do Semi- \\ árido (UFERSA) \\ luizjunior05@gmail.com
}

\begin{abstract}
ResumoUm ambiente de aprendizagem móvel provê aos estudantes uma forma de ensino não possível de ser realizada em um curso convencional baseado na web. O uso de Objetos de Aprendizagem $(\mathrm{OAs})$ em um formato padrão consiste em uma forma efetiva de permitir, entre outras características, o reuso e a interoperabilidade de conteúdo entre diferentes Sistemas de Gestão de Aprendizagem. No entanto, um problema que ocorre frequentemente é a inadequação do conteúdo sendo aprendido ao contexto do usuário. Um ambiente de suporte à aprendizagem móvel sensivel ao contexto pode resolver esse problema. Desta forma, este artigo apresenta uma abordagem baseada em agentes para recomendação sensível ao contexto de OAs a fim de aperfeiçoar o processo de ensino na aprendizagem móvel.
\end{abstract}

Palavras-Chave: Aprendizagem Móvel, Aprendizagem Ubíqua, Agente, Sensibilidade ao Contexto, Algoritmo Genético.

\begin{abstract}
A mobile learning environment provides students with a teaching method that would be not possible to be performed in a conventional web-based course. The use of Learning Objects (LOs) in a standard way consists of an effective way to allow, among other features, content reuse and interoperability among different Learning Management Systems. However, a problem that occurs frequently is the unsuitability of the content being learned to the context in which the student is inserted. A context-aware mobile learning environment allows to solve this problem. Thus, this paper presents an agent-based approach to context-aware recommendation of LOs in order to enhance the teaching process in mobile learning.
\end{abstract}

Keywords: Mobile Learning, Ubiquitous Learning, Agent, Context-awareness, Genetic Algorithm. 


\section{Introdução}

A Educação a Distância (EaD) é uma modalidade de ensino e aprendizagem que vem crescendo há alguns anos. De acordo com pesquisa realizada pela Associação Brasileira de Educação a Distância (ABED), 11\% do total de usuários de Internet no Brasil a utilizam também para fins educacionais, mais especificamente para cursos online [1].

Uma das formas de se prover a EaD é através do uso de dispositivos móveis, modalidade esta conhecida como Aprendizagem Móvel, do inglês Mobile Learning ou mlearning. Este meio de oferecer ensino permite que estudantes e professores possam tirar vantagens dos recursos oferecidos pelas tecnologias móveis, dentre os quais destaca-se a possibilidade de acessar, visualizar e prover conteúdo independentemente do horário e a partir de qualquer localidade [2].

Contudo, para melhorar a eficácia da absorção de conhecimento pelos estudantes na aprendizagem móvel, deve-se levar em consideração as características particulares de cada estudante. Isso é necessário não apenas para fornecer um conteúdo que atenda às características cognitivas dos estudantes, mas também para prover conteúdos de forma adequada às restrições dos seus dispositivos móveis, uma vez que os mesmos possuem recursos distintos e limitados (com restrições). Surge então o conceito de ambientes sensíveis ao contexto (context-aware environments). Esse tipo de ambiente se adéqua ao perfil do usuário, levando em consideração informações fornecidas pelo próprio usuário, além daquelas captadas dinamicamente a partir de sua interação com os dispositivos computacionais [3].

Ao considerarmos as características da aprendizagem móvel e a capacidade de detectar as informações de contexto do estudante, a fim de fornecer conteúdo educacional de forma personalizada, surge o conceito de Aprendizagem Ubíqua (também conhecida como u-learning, de ubiquitous learning) [23].

A fim de desenvolver ambientes de aprendizagem que sejam sensíveis ao contexto do estudante, é fundamental que os conteúdos educacionais sejam criados de maneira padronizada. Desta forma, é possível que um ambiente de suporte à aprendizagem exiba o conteúdo de forma adequada e reutilize conteúdos em diferentes contextos e a partir de diversos repositórios. Além disso, é possível combinar conteúdos distintos, o que, por sua vez, melhora o processo de produção de conteúdo e, como consequência, reduz os seus custos [4]. Um modo eficaz de padronizar conteúdos educacionais é através do uso de Objetos de Aprendizagem (OAs), os quais consistem em pequenas unidades de conteúdo que podem ser usadas, reutilizadas e referenciadas durante um processo de aprendizagem [5].

Este artigo apresenta um ambiente de aprendizagem móvel que, através do uso de OAs, ontologias, agentes de software e aplicação de um Algoritmo Genético (AG) [6] se adéqua às necessidades dos estudantes, fornecendo conteúdos educacionais (OAs) adequados às características do contexto no qual os mesmos se encontram.

Este artigo está dividido em dez seções. A Seção 2 mostra uma visão geral de sistemas multiagente. A Seção 3 descreve os objetos de aprendizagem, assim como os padrões usados na sua criação. A Seção 4 apresenta a técnica de Algoritmos Genéticos (AG). A Seção 5 traz conceitos relacionados a ambientes sensíveis ao contexto. A Seção 6 apresenta o conceito de aprendizagem ubíqua. Na Seção 7 são discutidos sistemas de recomendação. A Seção 8 discute os trabalhos relacionados. A Seção 9 descreve a abordagem baseada em agentes proposta neste artigo. A última seção apresenta nossas considerações finais e uma discussão sobre trabalhos futuros.

\section{Sistemas Multiagente}

De acordo com Russel e Norvig [7], agentes são entidades de software autônomas que percebem seu ambiente por meio de sensores e que atuam sobre esse ambiente através de atuadores, processando informação e conhecimento. Um Sistema Multiagente (SMA), por sua vez, consiste de um conjunto de agentes autônomos que colaboram para resolver um problema o qual seria impossível solucionar com apenas um agente.

Agentes podem ser construídos de várias formas. Podem ser agentes de software ou hardware, estáticos ou móveis, persistentes ou não-persistentes, reativos ou cognitivos (inteligentes) [8,9]. De acordo com Pontes [8], uma das classificações mais importantes de agentes é em relação a eles serem reativos ou cognitivos.

Agentes reativos são agentes que selecionam ações a serem executadas com base exclusivamente na percepção atual, não levando em consideração o histórico de percepções. Uma vez que não possuem memória, são incapazes de planejar ações futuras [7,8].

Por outro lado, os agentes cognitivos são mais complexos, pois possuem uma representação explícita tanto do ambiente quanto de outros agentes. Este tipo de agente possui uma memória interna, o que possibilita planejar ações futuras com base em situações que ocorreram anteriormente $[7,8]$.

Agentes de software podem realizar diversas tarefas em ambientes de aprendizagem, tais como monitorar as atividades do estudante no ambiente de aprendizagem, capturar de forma automática as informações de contexto dinâmico do estudante, recomendar conteúdos de interes- 
se deste, entre outras. Diante do aumento no número de estudantes que interagem com os sistemas de suporte à aprendizagem, o uso de agentes para realizar estas tarefas torna-se extremamente importante, principalmente devido ao fato de serem tarefas complexas para os professores gerenciarem a distância.

\subsection{Arquiteturas de Sistemas Multiagente}

Uma arquitetura bastante simples e que é muito utilizada no controle dos agentes é a arquitetura "Quadro Negro". Nessa arquitetura não é necessária uma comunicação direta entre os agentes, pois todas as comunicações são feitas por meio de uma estrutura de dados central (quadro negro) que é compartilhada por todos os agentes, sendo também responsável por controlar o acesso dos agentes. Quando se utiliza essa arquitetura, agentes podem escrever informações no quadro, assim como podem ler informações deixadas por outros agentes [13]. Essa será a abordagem adotada para a arquitetura do ambiente multiagente proposto neste trabalho.

Pode-se adotar também uma estratégia oposta ao quadro negro, na qual os agentes se comuniquem diretamente. Porém, esta abordagem acaba retirando um pouco da flexibilidade da arquitetura multiagente, pois obriga os agentes a possuírem uma identificação precisa, como um nome único no sistema, além da adoção de algum protocolo de comunicação entre eles [13].

A arquitetura adotada neste trabalho é do tipo Quadro Negro e será detalhada na Seção 9.

\subsection{Organização dos Sistemas Multiagente}

Os sistemas multiagente podem ser organizados de diversas maneiras, sendo três os tipos mais comuns [13]:

- Hierárquica: existe um agente superior que controla e toma as decisões, comunicando sua decisão para os demais agentes, que pertencem a um nível inferior na hierarquia;

- Comunidade de especialistas: todos os agentes estão em um mesmo nível, tendo cada agente uma especialidade em certo domínio. Nesse tipo de organização, a interação entre os agentes ocorre de acordo com regras previamente estabelecidas;

- Comunidade científica: os problemas são solucionados localmente e, em seguida, essas soluções são testadas e refinadas por agentes solucionadores de problemas.

A organização do sistema multiagente proposto neste trabalho é do tipo comunidade de especialistas. Na Seção
9, serão apresentados os quatro tipos de agentes especialistas que compõem a abordagem proposta.

\section{Objetos de Aprendizagem}

Um conceito relevante quanto ao conteúdo didático utilizado em EaD é o de Objeto de Aprendizagem (OA). Segundo o Comitê de Padronização de Tecnologias de Aprendizagem (LTSC - Learning Technology Standard Comitee), do Institute of Electrical and Electronics Engineers (IEEE), um OA é uma entidade material educacional, digital ou não, que pode ser usada para aprendizagem, educação ou treinamento [5]. Dessa forma, podem ser elencadas quatro propriedades principais dos OAs: reusabilidade, acessibilidade, interoperabilidade e durabilidade [10].

Em resumo, a ideia central do conceito de OAs é permitir que os projetistas educacionais construam componentes educativos relativamente pequenos que possam ser usados em diferentes contextos de aprendizagem. Ou seja, são conteúdos digitais que, além de permitir alcançar um objetivo educacional, promovem a reusabilidade dos conteúdos utilizados no processo de ensino-aprendizagem.

No entanto, para garantir os benefícios em se utilizar OAs, há de se considerar certas características na criação de OAs digitais, como, por exemplo:

- definição da estrutura de navegação;

- adequação do conteúdo de uma mídia escrita para uma mídia digital;

- atendimento aos aspectos pedagógicos de ensino;

- integração do OA com diferentes tipos de ambientes de $\mathrm{EaD}[4]$.

Nesse sentido, utiliza-se padrões de desenvolvimento de OAs para que estes possam garantir as quatro propriedades citadas anteriormente [11].

\subsection{Padrões de Objetos de Aprendizagem}

Segundo [11], padrões de OAs constituem um meio de organizar os dados de um OA para prover comunicação entre diferentes ambientes computacionais, bem como garantir seu acesso e usabilidade, além de prover interoperabilidade. Os autores relatam que esses padrões são divididos, de acordo com suas funcionalidades, em: padrões de metadados, de empacotamento, de interface e comunicação e de integração.

Para o presente trabalho, é importante descrever as características dos padrões de integração. Esse tipo de padrão, como o próprio nome indica, unifica em um modelo de referência diferentes tipos de padrões, tais como padrões de metadados, empacotamento, interface e comuni- 
cação, desenvolvidos por outras organizações [11]. O padrão de integração SCORM (Sharabale Content Object Reference Model), desenvolvido pela ADL (Advanced Distributed Learning) [10], integra um conjunto de padrões técnicos, especificações e orientações designadas a atender os requisitos de alto nível do SCORM - sistemas e conteúdo acessíveis, interoperáveis, duráveis e reutilizáveis. O conteúdo no padrão SCORM pode ser distribuído para os estudantes através de qualquer Sistema Gestão de Aprendizagem (LMS - Learning Management System), que seja compatível com o SCORM e que use a mesma versão deste [10].

Neste trabalho utilizaremos OAs desenvolvidos segundo o padrão SCORM, tanto pelo fato de ser um padrão amplamente difundido, como também por ser composto de um conjunto de outros padrões. Desta forma, podemos usufruir das melhores características de cada padrão.

\section{Algoritmos Genéticos}

Para entender o que são algoritmos genéticos é preciso inicialmente definir o que são algoritmos evolucionários. Estes são algoritmos que usam modelos computacionais dos processos naturais de evolução como uma ferramenta para resolver problemas [6]. Mesmo existindo uma grande variedade de modelos computacionais propostos, todos eles possuem o mesmo princípio básico: todos simulam a evolução das espécies baseando-se na teoria da evolução humana e utilizando os denominados operadores genéticos $[6,12]$.

Os AGs constituem um ramo dos algoritmos evolucionários e, portanto, também podem ser definidos como uma metáfora do processo biológico de evolução natural [6]. De uma forma sucinta, os AGs tentam resolver problemas para os quais não existe um algoritmo conhecido, gerando-se uma população inicial e, de acordo com critérios de avaliação, selecionando os melhores indivíduos dessa população, que servirão como solução para o problema ou, caso contrário, serão combinados para obter uma nova geração. Esse processo é repetido até que se encontre uma solução ou até que se perceba que não serão alcançadas melhores soluções nas novas gerações [13].

Os AGs, assim como os demais tipos de algoritmos evolucionários, não podem garantir que uma geração será melhor do que a sua geração antecessora, mesmo sendo pouco provável não ocorrer tal situação. Isso acontece devido ao fato desses algoritmos serem baseados na evolução natural que, por sua vez, não consiste em um processo dirigido à obtenção da solução ótima. De fato, o processo simplesmente consiste em fazer competir um conjunto de indivíduos, fazendo com que sobrevivam aqueles indivíduos que são mais aptos $[6,12]$.
O trabalho proposto utiliza um AG para recomendar OAs que sejam adequados às características e preferências de determinado estudante, tentando sempre chegar ao melhor conjunto a ser recomendado. Para um melhor entendimento da solução proposta, as próximas subseções descrevem os principais componentes de um AG.

\subsection{Operadores Genéticos}

Operadores genéticos podem ser definidos, de uma forma concisa, como aproximações computacionais de fenômenos vistos na natureza, tais como reprodução sexuada (exige a presença de dois organismos, na maioria das vezes de sexos opostos, que trocam material genético), mutação genética, seleção de indivíduos, além de outros processos que podem ser criados pelos próprios programadores. A ocorrência de tais processos depende do "desempenho" dos indivíduos desta espécie dentro do "ambiente" [6]. Ou seja, é necessário que um indivíduo mostre características que lhe atribuam destaque dentro do grupo para que ele possa, consequentemente, sobreviver para uma próxima geração.

Após a seleção dos indivíduos em uma população, são aplicados os operadores genéticos, obtendo-se assim uma população com melhores indivíduos ou não. Esse procedimento é repetido através de sucessivas gerações até que se atinja um resultado satisfatório [14]. Os AGs básicos são geralmente constituídos de dois operadores: cruzamento e mutação [14].

\subsubsection{Cruzamento (Crossover)}

Usando-se este operador, ocorre o cruzamento entre o material genético de dois indivíduos pais, combinando informações de modo que exista uma probabilidade razoável dos novos indivíduos produzidos serem melhores que seus pais [14].

Depois de selecionados dois pais de acordo com o módulo de seleção escolhido, um ou mais pontos de corte são selecionados aleatoriamente, de acordo com a estratégia adotada. Um ponto de corte consiste em uma posição entre dois genes de um cromossomo [13].

A taxa de cruzamento $\left(\rho_{\mathrm{c}}\right)$ é um parâmetro de controle que deve ser levado em consideração quando da construção de um AG. Este parâmetro indica a probabilidade de ocorrência do cruzamento entre os indivíduos selecionados na população. É consenso entre os estudiosos da área que a probabilidade de ocorrência do cruzamento deve ser alta, fazendo com que $\rho_{\mathrm{c}}$ pertença geralmente ao intervalo $[0,6 ; 0,9][13]$. Quanto maior a taxa, mais rapidamente novas estruturas serão introduzidas na população. Porém, deve-se tomar certo cuidado, pois uma taxa muito alta poderá eliminar rapidamente indivíduos de boa qualidade da população [14]. 
Apesar de ser pouco provável, pode acontecer, em último caso, de não ocorrer o cruzamento entre os pais selecionados, caso no qual os filhos serão cópias exatas dos pais [13].

\subsubsection{Mutação}

Depois de compostos os filhos, pode ser utilizado o operador de mutação, o qual modifica aleatoriamente o código genético destes, atribuindo a eles características que não são pertencentes aos pais e, consequentemente, cria uma variabilidade maior entre os descendentes. Caso esteja sendo utilizada uma codificação binária, alguns bits podem ser invertidos. Caso seja uma codificação real ou inteira, pequenos valores podem ser somados aleatoriamente [13].

Um parâmetro que deve ser considerado é a taxa de mutação $\left(\rho_{\mathrm{m}}\right)$, que corresponde à probabilidade de ocorrência da mutação nos indivíduos da população no decorrer do processo de evolução. Uma alta taxa de busca possibilita uma maior varredura do espaço de busca. Por outro lado, caso $\rho_{\mathrm{m}}$ seja muito alta, pode tornar a busca aleatória, como uma técnica de random walk [6]. É consenso que a probabilidade de ocorrência da mutação é extremamente baixa, devendo o valor de $\rho_{\mathrm{m}}$ ser da ordem de $0,5 \%$ [6] a $1 \%[13]$.

\section{Ambientes Sensíveis ao Contexto}

Sensibilidade ao contexto descreve um paradigma no qual o contexto de um usuário é levado em consideração para definir o seu perfil. Não existe um consenso a respeito da definição de "contexto", sendo este específico da aplicação e da intenção desejadas, requerendo a identificação das funções e propriedades dos domínios dos indivíduos $[3,15]$.

O contexto pode ser definido de acordo com informações relativas a propriedades que se combinam para descrever e caracterizar uma entidade e seu papel de uma forma compreensível pelo computador $[3,14]$. A localização do estudante, por exemplo, é uma característica importante para a definição do seu contexto em um ambiente para aprendizagem móvel. Entretanto, o contexto inclui mais do que apenas a localização. De fato, quase todas as informações disponíveis no momento da interação podem ser vistas como informações contextuais, dentre as quais destacam-se $[3,16]$ :

(i) as diversas tarefas exigidas dos usuários;

(ii) a variada gama de dispositivos que se combinam para criar sistemas móveis, com a infraestrutura de serviços associada;

(iii) disponibilidade de recursos (ex. condição da ba- teria, tamanho de tela, etc.);

(iv) situação física (ex. nível de ruído, temperatura, nível de luminosidade etc.);

(v) informação espacial (ex. localização, velocidade, orientação etc.); e

(vi) informação temporal (ex. hora do dia, data etc.).

Esta lista, embora não contenha exatamente todas as informações que podem ser consideradas, serve para demonstrar a complexidade inerente ao contexto, sua natureza de domínio específico e a dificuldade em defini-lo e medi-lo [3]. Na tentativa de facilitar a compreensão sobre o contexto, Moore et al. [15] definem dois tipos gerais de contexto: estático (denominado customização) e dinâmico (denominado personalização). $\mathrm{O}$ primeiro diz respeito à situação na qual um perfil de usuário é criado manualmente, estando o usuário ativamente envolvido no processo e tendo um elemento de controle. Já o contexto dinâmico refere-se à situação na qual o usuário é visto como sendo passivo, ou pelo menos com um pouco menos de controle no processo de criação de seu perfil. Nesse caso, o sistema monitora, analisa e reage dinamicamente ao comportamento do usuário e ao perfil identificado.

Algumas aplicações de aprendizagem móvel sensíveis ao contexto utilizam contextos de aprendizagem a fim de adaptar ou sugerir apropriadamente atividades e conteúdos [2]. Entretanto, os trabalhos encontrados na literatura não levam em consideração os recursos físicos dos dispositivos móveis, o que compromete uma definição mais precisa do contexto dos estudantes e, consequentemente, o acesso e navegação adequados nos conteúdos recomendados, uma vez que isto é diretamente influenciado pelas características dos dispositivos móveis utilizados pelos estudantes.

A proposta apresentada neste artigo, além de considerar as características contextuais dos estudantes, leva em consideração para recomendação as características dos dispositivos móveis que estes utilizam.

\subsection{Uso de Ontologias em Ambientes Sensí- veis ao Contexto}

Formalmente, uma ontologia pode ser considerada como sendo uma especificação formal explícita conceitualizando um domínio de interesse específico com estrutura e detalhe semânticos para representar conceitos, entidades, atributos, relacionamento, restrições, axiomas e suas propriedades de uma forma legível para o humano e para o computador $[3,15]$.

A partir desse conceito, é possível perceber que uma ontologia é uma "especificação de um conceito", ou seja, ela é utilizada para especificar um conhecimento a respei- 
to de um determinado domínio de conhecimento. Isso significa que uma ontologia permite a um projetista especificar, de uma forma aberta e significativa, os conceitos e relacionamentos que caracterizam de modo coletivo algum domínio. A vantagem de se utilizar uma ontologia é que, mesmo sendo desenvolvida com uma finalidade específica, ela pode ser publicada e reutilizada para outros propósitos [30]. Por exemplo, uma vinícola pode usar a ontologia de vinho para ligar sua programação de produção ao sistema de estoque no armazém do vinho.

Há muitas formas de escrever uma ontologia e uma variedade de opiniões sobre que tipos de definição devem englobar. Porém, na prática, os conceitos de uma ontologia são largamente dirigidos pelos tipos de aplicação que elas terão de suportar [30].

Visto que são formas de representar o conhecimento de um dado domínio, as ontologias podem ser usadas em ambientes de aprendizagem para representação de conceitos e modelos inerentes ao ambiente em questão [8]. Por exemplo, em [31] é descrita uma ontologia para apoiar a construção de ambientes interativos de aprendizagem. Utilizando-se de uma ontologia, são modelados diversos conceitos de um ambiente de aprendizagem, como: modelo do estudante, que representa o estudante que será ensinado; modelo pedagógico, que diz respeito às estratégias para aprendizagem; modelo de colaboração, que define a forma de colaboração no ambiente; e modelo de domínio, que refere-se ao que será ensinado.

As ontologias podem ser utilizadas com diversas finalidades em ambientes de aprendizagem, sendo uma das aplicações desta tecnologia a personalização de ambientes de aprendizagem. Isso é alcançado levando-se em consideração características específicas do perfil de cada estudante. Em [32], é proposto um framework para adaptação em ambientes de aprendizagem, no qual dois modelos ontológicos são construídos: um modelo de conhecimento, no qual são guardadas as informações relacionadas ao objetivo do estudante, e um modelo do estudante, o qual armazena, além do seu objetivo, outras informações, tais como histórico de aprendizagem, conceitos dominados e grau de desempenho. Dessa forma, com base no modelo do estudante armazenado em uma ontologia, o sistema irá se adaptar de forma a cumprir os requisitos do estudante.

Em [32], é apresentada uma abordagem para personalização de aprendizagem apoiada por dispositivos computacionais com base em ontologia, na qual a troca de informações é mantida por serviços Web (web services), sob uma arquitetura orientada a serviços. A ideia principal do sistema é identificar requisitos do usuário, ou seja, as preferências dos estudantes e suas características, e criar o modelo do estudante. Nesse modelo constará o seu conhecimento expresso através de um conjunto de palavras-chave pertencentes a uma ontologia comum para, de acordo com o modelo de cada estudante, adaptar o curso de forma individual.

Diante dos trabalhos apresentados, é possível perceber a variedade de finalidades com as quais as ontologias podem ser utilizadas em ambientes sensíveis ao contexto. No presente trabalho, ontologias são utilizadas para modelar tanto o perfil estático quanto o perfil dinâmico dos estudantes, conforme será descrito na Seção 9.

\section{Aprendizagem Ubíqua}

Mark Weiser [24] idealizou o mundo da computação ubíqua, onde o computador deixa de ser usado como um dispositivo de propósito geral, passando a ter uma nova visão de sua funcionalidade, atendendo as necessidades específicas de cada usuário. Neste cenário, um usuário não se restringe a acessar um único computador, mas vários dispositivos computacionais interligados entre si e disseminados de forma quase imperceptível pelo ambiente.

A partir do conceito apresentado, pode-se definir a aprendizagem ubíqua como sendo a utilização de dispositivos móveis, tecnologias de comunicação móvel sem fio, sensores e mecanismo de localização com o objetivo de auxiliar o processo educacional, levando em consideração características particulares dos estudantes [23].

Desta forma, a aprendizagem ubíqua pode ser considerada como sendo a aprendizagem móvel que é realizada levando-se em consideração as características do contexto dos estudantes, provendo a estes conteúdos adaptados às suas necessidades [25]. Assim, a aprendizagem ubíqua provê um novo paradigma, através do uso de dispositivos móveis, que fornece um serviço de forma transparente aos estudantes [26].

A mobilidade permitida ao usuário devido ao uso de dispositivos móveis em aplicações ubíquas torna ainda mais importante a consideração do contexto de um estudante, visto que as características do estudante podem se modificar a qualquer momento. Essas modificações podem ocorrer em diversos aspectos (ex. condições físicas, recursos físicos disponíveis, recursos computacionais etc.) [26].

Este artigo apresenta uma aplicação para aprendizagem ubíqua onde a mobilidade é provida pelo uso de dispositivos móveis e agentes de software e ontologias provêem a sensibilidade ao contexto do estudante.

\section{Sistemas de Recomendação de OAs}

De acordo com Vieira e Nunes [27], o aumento de meios de disponibilização de conteúdo, podendo ser produtos ou informação, através de sistemas Web, provoca 
uma situação onde o usuário possui muitas opções de escolha antes mesmo de estar apto a selecionar uma opção que atenda suas necessidades. Os Sistemas de Recomendação (SR) buscam amenizar os impactos gerados por essa sobrecarga de informação.

Os SRs utilizam repositórios de informação e dados de preferência dos usuários para direcionar conteúdos aos indivíduos com potenciais interesses. Um dos desafios dos SRs é realizar a indicação de produtos, serviços e/ou informação que melhor atendam as expectativas dos usuários (e ao seu perfil) [28].

Segundo Vieira e Nunes [27], os SRs podem ser classificados em três tipos: Sistema de Recomendação Baseada em Conteúdo (SRBC), Sistema de Recomendação Colaborativa (SRC) e Sistema de Recomendação Híbrida (SRH). A recomendação baseada em conteúdo é realiza através de informações recuperadas do usuário (perfil, comportamento) e/ou do seu histórico de escolhas. Já a recomendação por filtragem colaborativa leva em consideração escolhas realizadas por usuários com características similares. Por último, o SRH efetua a recomendação através da aplicação das técnicas utilizadas pelos SRBC e SRC, de forma a atingir um maior número de possibilidades e sugerir objetos que diretamente não aparentam estar relacionados [29].

No âmbito da educação ubíqua, os alunos são expostos a uma grande quantidade de OAs e podem levar um bom tempo para realizar escolhas difíceis. Tendo em vista que os estudantes estão em processo de formação, estes não estão aptos o suficiente para realizar tais escolhas. Um SR vem a suprir esta carência recomendando OAs apropriados e tirando a responsabilidade de escolha do estudante.

O SR utilizado no presente trabalho é um SRH, visto que utiliza tanto recomendação baseada em conteúdo, analisando os metadados dos OAs no padrão SCORM, como também recomendação colaborativa, uma vez que considera as escolhas de outros estudantes.

\section{Trabalhos Relacionados}

A utilização de sistemas de recomendação em ambientes educacionais não é nenhuma novidade. Em Primo et al. [17] é apresentado um modelo para a recomendação de conteúdos educacionais descritos através de metadados. Esse modelo considera perfis de usuários e interoperabilidade entre aplicações educacionais, além de aspectos cognitivos de aprendizado. Esse trabalho também apresenta como conteúdos educacionais podem ser descritos através de ontologias, o que facilita também a inferência dos conteúdos apropriados aos perfis dos usuários.

Em Gluz \& Vicari [18] é apresentada a MILOS (Mul- tiagent Infrastructure for Learning Object Support), uma infraestrutura, combinando ontologias e agentes, que implementa as funcionalidades necessárias aos processos de autoria, gerência, busca e disponibilização de OAs compatíveis com a proposta de padrão de metadados de OAs OBAA (Objetos de Aprendizagem Baseados em Agentes).

Yau \& Joy [2] propõem uma arquitetura de aprendizagem móvel sensível ao contexto composta por três componentes principais: o perfil do estudante, um mecanismo de personalização e um repositório de OAs. O perfil do estudante serve para armazenar suas preferências de aprendizagem móvel, capturadas através de um questionário respondido pelo estudante. $\mathrm{O}$ mecanismo de personalização recebe esse perfil e combina as informações dele com aquelas obtidas dinamicamente através de sua interação com o ambiente. Em seguida, o mecanismo de personalização compara todas as informações obtidas com os metadados dos OAs disponíveis no repositório. O sistema então recomenda OAs adequados aos estudantes de acordo com as características do seu contexto.

O presente trabalho reúne as principais características dos trabalhos citados anteriormente. Porém, o fator que difere o presente trabalho dos demais é o fato dele utilizar $\mathrm{AG}$ para recomendar OAs adequados às diversas características do contexto do estudante, inclusive os recursos dos dispositivos móveis, os quais podem constituir um fator limitante na aprendizagem do estudante no momento da recomendação dos OAs. Pelo fato de ser projetado para dispositivos móveis, o ambiente provê ao estudante flexibilidade quanto às opções de estudo. Utilizando agentes de software em combinação com as ontologias de descrição dos perfis (estático e dinâmico) dos estudantes e o AG, o ambiente recomenda, de maneira autônoma, conteúdos educacionais adequados às características pessoais e cognitivas dos estudantes.

\section{Abordagem Baseada em Algoritmo Genético para Recomendação de Objetos de Aprendizagem Sensível ao Contexto do Estudante}

A arquitetura do ambiente proposto é apresentada na Figura 1. Como pode ser visto na Figura 1, os estudantes devem, inicialmente, se autenticar no LMS e acessar algum dos cursos nos quais esteja matriculado. Para isto, eles precisam realizar um cadastro no qual irão informar algumas características de seu perfil, como, por exemplo, local e hora do dia preferidos para o estudo, sua área de interesse, entre outras. Essas informações servirão para a instanciação de um componente essencial da arquitetura, que é a ontologia de contexto estático dos estudantes. 




Figura 1. Arquitetura da solução proposta.

Os agentes descritos nesta abordagem foram desenvolvidos utilizando o JADE (Java Agent Development Framework), que consiste em uma plataforma completa para desenvolvimento e execução de SMA [19]. Também foi utilizado o framework de desenvolvimento de aplicações MLE (Mobile Learning Engine) [20] para a construção do ambiente.

Outro componente de extrema relevância é o repositório de OAs. Para que o mecanismo de recomendação funcione, é necessário que estes OAs estejam em conformidade com o padrão SCORM. Através dos metadados descritos nesse padrão, o agente será capaz de comparar as informações dos OAs com os perfis dos estudantes e realizar a recomendação de OAs de maneira adequada.

A organização do SMA é do tipo comunidade de especialistas, pois cada um dos quatro tipos de agentes criados para este trabalho encontra-se no mesmo nível, sendo cada um deles especialista em determinada tarefa. Os agentes interagem entre si através de um protocolo de comunicação previamente estabelecido. Para o desenvolvimento dos agentes, foram utilizadas as bibliotecas do JADE e os mesmos executam sob esta plataforma.

\subsection{Agentes de Software}

Como é possível perceber na Figura 1, o SMA é composto de quatro tipos de agentes: Agente Estudante (Student Agent - SAg), Agente Recomendador (Recommender Agent - RAg), Agente de Interface (Interface Agent - IAg) e Agente DF (Directory Facilitator).

$\mathrm{O}$ agente DF possui o comportamento voltado para a mediação da comunicação entre os outros agentes. Sua função principal é fornecer uma arquitetura do tipo "quadro-negro", onde agentes escrevem informações, procuram por informações escritas por outros agentes e conseguem, através do serviço provido pelo DF, se comunicar com o agente que escreveu aquela informação.

Os SAgs são responsáveis por monitorar as atividades dos estudantes, atualizar e recuperar, das ontologias de contexto estático e dinâmico, as preferências de aprendi- zagem que compõem os perfis dos estudantes e seus respectivos históricos de escolhas de OAs. Os SAgs também capturam as informações de localização geográfica e do horário corrente. Ou seja, o comportamento do SAg é voltado para monitoramento das informações relativas ao estudante. Em seguida, todas essas informações são cadastradas no agente DF.

O RAg tem o intuito de detectar OAs adequados ao contexto do estudante, levando em consideração:

(i) as informações consultadas no agente DF;

(ii) as informações dos OAs disponíveis no repositório de OAs SCORM; e

(iii) as preferências similares de outros estudantes. Após aplicar o AG para identificar os OAs adequados, o RAg cadastra, no agente DF, as novas informações, as quais servirão ao IAg.

A Figura 2 mostra a interface principal do ambiente e uma mensagem de recomendação de OAs efetuada pelo RAg.

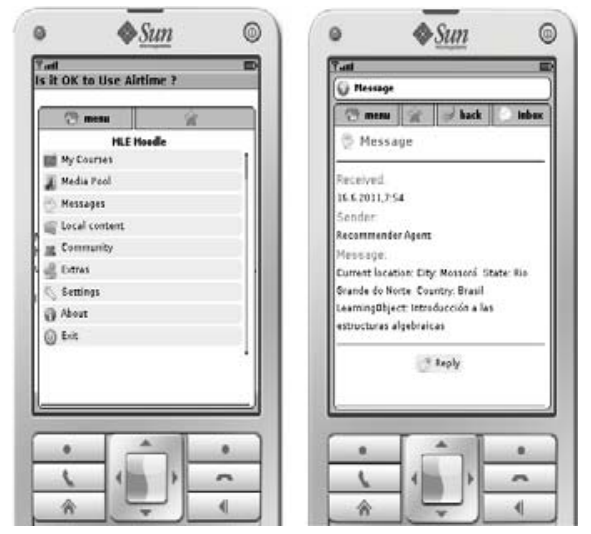

Figura 2. Tela inicial e sugestão de OAs.

O IAg é responsável, principalmente, por verificar a adequação visual do OA recomendado pelo RAg às características do dispositivo móvel do estudante. Caso o OA não seja adequado ao dispositivo, o IAg rejeitará a recomendação. Além disso, o IAg tem a responsabilidade de informar ao professor o conteúdo que foi sugerido. $\mathrm{O}$ diagrama de sequência da Figura 3 mostra o fluxo de troca de mensagens entre esses agentes através do modelo de interação. 


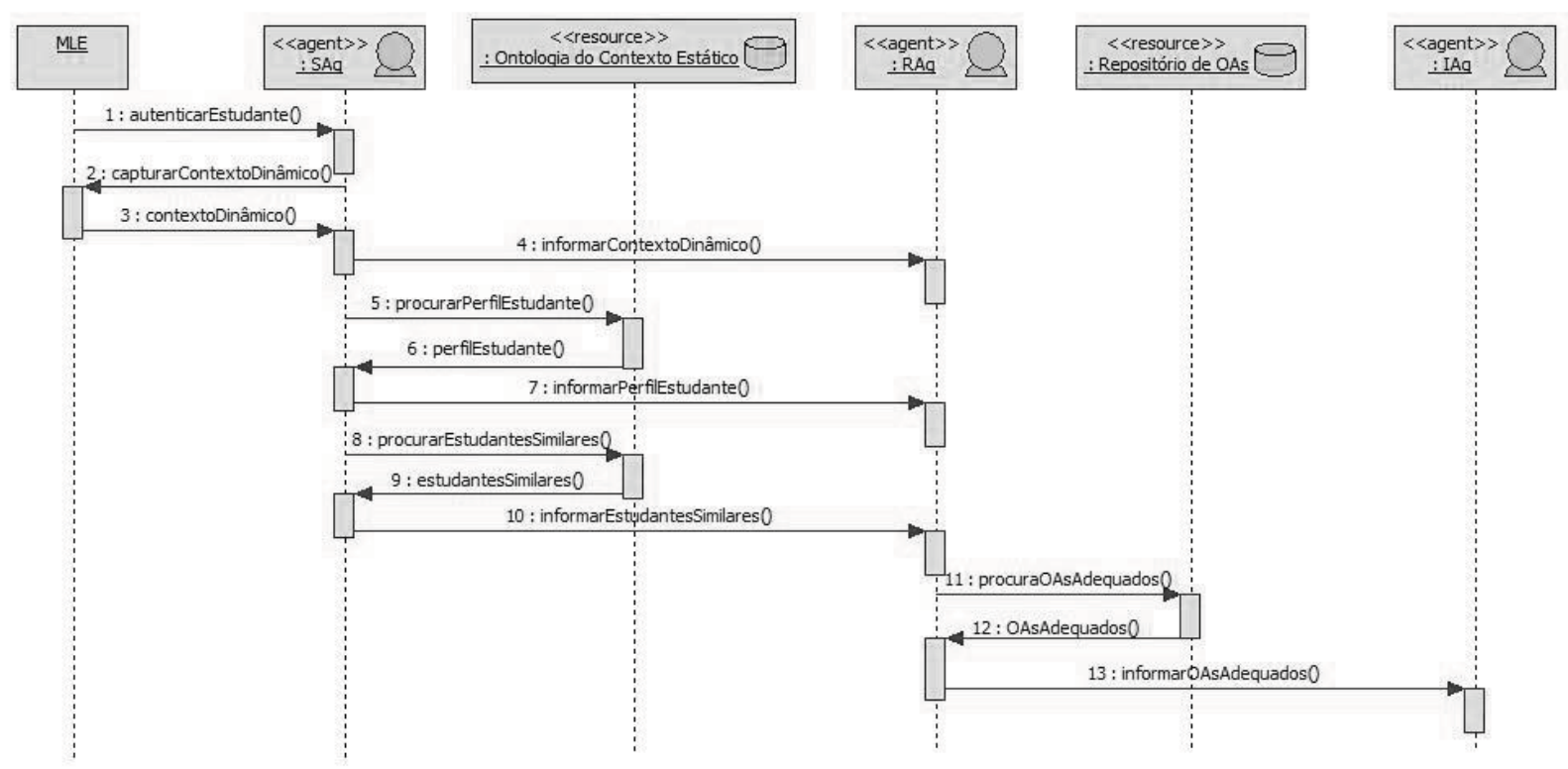

Figura 3: Modelo de Interação entre os Agentes.

(ii) Afinidade com o dispositivo móvel: determina o

\subsection{Recomendação Sensível ao Contexto}

A identificação dos OAs que serão recomendados é realizada pelo RAg, através da aplicação de um AG, o qual será melhor descrito a partir da Subseção 9.3. A utilização do AG é justificada pela alta complexidade do problema de recomendação, o que pode denegrir o desempenho do SMA quando o número de OAs for suficientemente grande. Neste caso, um algoritmo aproximativo (AG) viabiliza a busca por uma solução próxima da ótima.

Para realizar uma recomendação de OAs adequada ao contexto do estudante, é essencial levar em consideração características que podem limitar a aprendizagem do mesmo.

Na modelagem do problema no AG, inicialmente, temos uma população composta de certa quantidade de OAs. Cada OA possui uma série de características que o relaciona com o contexto do estudante. No presente trabalho, foram consideradas as características detalhadas a seguir:

(i) Afinidade com o curso: representa o grau de relacionamento do conteúdo de um OA a determinado curso. Esse grau de afinidade é representado no AG em uma escala que varia de 0 (nenhuma afinidade) a 5 (alta afinidade). Determinado OA pode estar relacionado a um ou vários cursos, sendo o administrador de cada curso responsável por determinar, manualmente, quais OAs são afins ao curso e qual o seu grau de afinidade. grau de adequação do conteúdo de um OA ao dispositivo móvel do estudante. O grau de afinidade com o dispositivo é definido no AG de acordo com uma faixa que varia de 0 (nenhum recurso suportado) a 5 (todos os recursos suportados). As informações do dispositivo são capturadas a partir da requisição HTTP enviada do dispositivo móvel para o servidor no qual se encontra o LMS. Em seguida, em posse dessas informações, é possível verificar em uma base de dados, chamada WURFL [21], quais recursos aquele dispositivo móvel suporta.

(iii) Horário de estudo: os horários de estudo são definidos em faixas de horários. $\mathrm{O}$ estudante define, inicialmente, qual o horário de estudo preferido dele. Assim, quando o estudante acessa o ambiente de aprendizagem, o sistema se encarrega de verificar se o horário corrente está incluso na faixa de estudo preferida do estudante. Caso esteja nessa faixa, o sistema atribui o maior valor possível a essa variável no AG (valor 5). Do contrário, será atribuído um valor cada vez menor, sendo o mínimo 1 , à medida que o horário corrente se distancie daquele definido como preferido pelo estudante.

(iv) Localização corrente: outra informação que pode ser levada em consideração para a definição do contexto do estudante é a localidade na qual ele se encontra. Em posse dessa informação, é possível verificar, nos metadados do padrão de OAs LOM (Learning Object Metadata) [5], a incidência de palavras relacionadas à localização do estudante. Com base nisso, são capturadas e consideradas informações do país, estado e cidade na qual o estudante se encontra. Quanto mais palavras relacionadas à locali- 
zação do estudante forem encontradas, maior será o valor atribuído a essa variável no AG (máximo 5). Por outro lado, caso não seja encontrada nenhuma informação relacionada, será atribuída a pontuação mínima (valor 0).

(v) Escolhas de outros estudantes: define um valor proporcional que representa a quantidade de acessos de um OA. A informação de quais OAs são mais acessados é levada em consideração em conjunto com a informação de afinidade do OA com o curso, pois de nada adiantaria para a recomendação um $\mathrm{OA}$ ser o mais acessado e não possuir nenhuma afinidade com o curso em questão. Desta forma, esse valor pode variar de 0 a 5 dependendo da quantidade de acessos e da sua relação com o curso.

(vi) Incidência de palavras-chave: os OAs disponibilizados no padrão SCORM possuem metadados que informam as palavras-chave relacionadas ao assunto daquele OA (tag keywords), além do título deste. Essas informações estão localizadas, mais especificamente, no padrão LOM, o qual é parte integrante do padrão SCORM. Em posse dessas informações, é feita uma verificação da incidência dessas palavras na descrição do curso do qual o estudante está participando. Quanto maior a quantidade de palavras-chave relacionadas, a probabilidade de que o OA possua assuntos relacionados ao curso será maior, sendo pontuado, portanto, com um peso maior (no máximo 5). Por outro lado, quanto menor a quantidade de palavras-chave relacionadas, o peso atribuído ao OA nessa variável no AG torna-se proporcionalmente menor.

\subsection{Projeto do Algoritmo Genético}

O projeto do AG é composto de classes auxiliares, responsáveis por capturar as informações, e do núcleo do AG, ou seja, das classes que efetivamente realizam a abordagem genética. O núcleo da abordagem baseada em AG criada para o presente trabalho foi codificada basi-

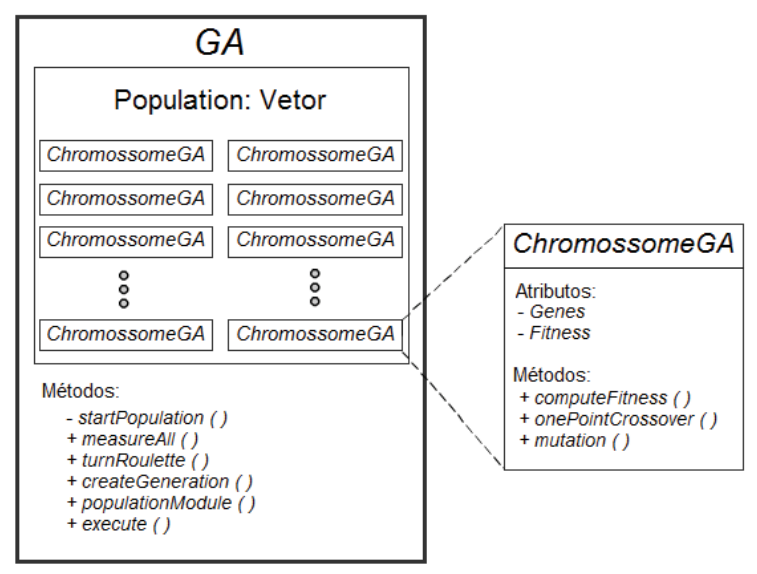

Figura 4: Estrutura e relacionamento entre as classes $G A$ e ChromossomeGA camente em duas classes:

- Classe $G A^{1}$, a qual consiste na representação do AG propriamente dito, contemplando todos os parâmetros necessários ao $\mathrm{AG}$.

- Classe ChromossomeGA, que representa as informações de cada um dos OAs no repositório e as operações que podem ser aplicadas sobre estes.

A Figura 4 mostra, de forma simplificada, os principais componentes dessas classes e como elas se relacionam entre si.

Como é possível perceber na Figura 4, várias instâncias da classe ChromossomeGA são utilizadas pela classe $G A$. As Subseções a seguir trazem uma descrição mais detalhada dessas classes.

\subsubsection{Classe $\boldsymbol{G} \boldsymbol{A}$}

A classe $G A$ possui a representação dos parâmetros necessários para a execução do AG. Alguns desses parâmetros são definidos como constantes, enquanto outros são passados através do construtor da classe no momento em que esta é instanciada pelo agente de software responsável (RAg). Dentre os principais parâmetros, destacamse os seguintes:

- Quantidade de gerações do AG: após a realização de uma série de testes, foi definido um total de cinquenta gerações para o AG criado.

- Informações do estudante: atributo que é uma instância da entidade Student, criada especificamente para guardar as informações do estudante. Esse objeto é manipulado pelos SAgs.

- Taxa de crossover $\left(\rho_{\mathrm{c}}\right)$ : o valor foi definido como $60 \%$ (ou 0,6).

- Taxa de mutação $\left(\rho_{\mathrm{m}}\right)$ : foi definido um valor de $5 \%$ (ou 0,05 ) para $\rho_{\mathrm{m}}$.

- Tamanho do cromossomo: essa informação será melhor detalhada na Subseção 9.4.

Além dos parâmetros citados, essa classe classe também engloba os métodos necessários para execução do $\mathrm{AG}$, dentre os quais cabe destacar:

- startPopulation: inicializa a população de cromossomos do AG.

\footnotetext{
Abreviação do termo, em inglês, Genetic Algorithm.
} 
$\mathrm{OA}(\mathrm{ID}+$ Fitness $=17$ bits $)$

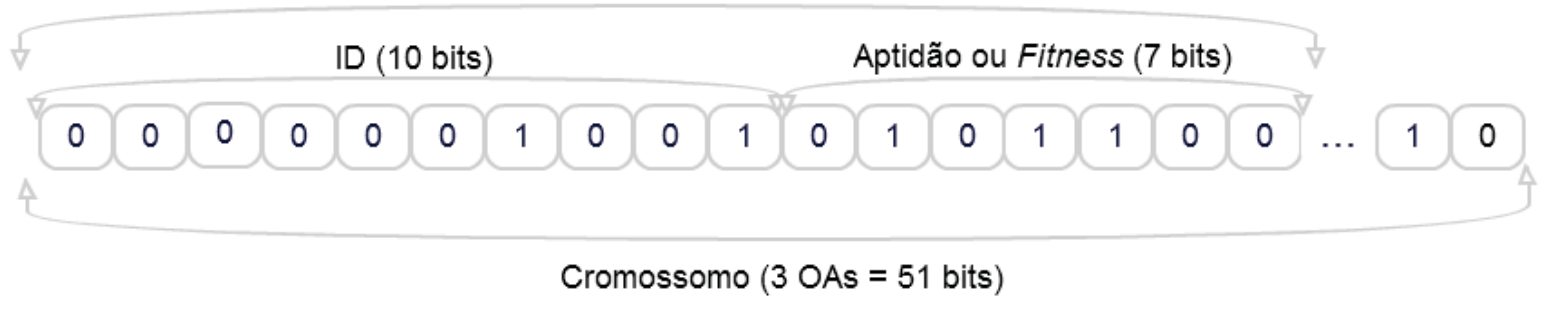

Figura 5. Representação cromossomial utilizada.

- measureAll: calcula a aptidão de todos os cromossomos e, ao final de sua execução, salva o resultado de todas as avaliações para auxiliar no método do giro da roleta.

- turnRoulette: implementa a técnica do giro da roleta, também conhecida como "Roleta Viciada" [6], para escolher os pais que participarão do próximo cruzamento.

- createGeneration: cria uma nova geração da população.

- populationModule: controla o tamanho da população, mantendo sempre o tamanho da população existente como sendo igual ao número de filhos gerados.

- execute: executa o AG de acordo com os parâmetros já instanciados e o número de gerações definido.

\subsubsection{Classe ChromossomeGA}

A classe ChromossomeGA possui as informações de cada um dos OAs pertencentes ao repositório e as operações que podem ser aplicadas sobre estes. As instâncias dessa classe são manipuladas pela classe GA. Merecem destaque os seguintes atributos da classe Chromossome$G A$ :

- Genes: cadeia de caracteres contendo todos os genes que formam um cromossomo.

- Fitness: valor que representa a aptidão de um OA em determinado contexto.

Dentre os métodos dessa classe, merecem destaque os seguintes:

- computeFitness: calcula a aptidão de cada um dos cromossomos individualmente.
- onePointCrossover: realiza o crossover de um ponto entre dois pais escolhidos através do método do giro da roleta.

- mutation: realiza a mutação dos filhos.

\subsection{Aspectos de Codificação do Algoritmo Genético}

As descrições das classes GA e ChromossomeGA fornecem uma visão de projeto para auxiliar no entendimento de como o AG foi criado. Entretanto, para conseguir uma melhor compreensão do AG, é necessário entender alguns aspectos mais detalhados relacionados à sua codificação. As Subseções 9.4.1, 9.4.2 e 9.4.3 discutem alguns aspectos importantes relacionados a esse assunto.

\subsubsection{Representação Cromossomial}

No AG cada cromossomo é formado por um conjunto de três OAs. A representação do OA (gene) é formada pelo ID e sua respectiva aptidão. O cálculo da aptidão do OA é o somatório dos valores atribuídos a cada característica citada na Subseção 9.2. Desta forma, cada cromossomo (ou indivíduo da população) é composto conforme o exemplo de cromossomo mostrado na Figura 5.

A população é composta de cromossomos que possuem, cada um, cinquenta e um bits em representação binária (0 ou 1). Cada OA é representado por dezessete bits, sendo dez utilizados para a sua identificação (ID) e sete para a representação do resultado de sua aptidão, considerando os aspectos descritos anteriormente. Assim, com cada OA utilizando dezessete bits, e com uma representação de cromossomo de cinquenta e um bits, é possível representar até três OAs por cromossomo (17 x $3=51$ bits).

A utilização da representação em codificação binária foi escolhida porque percebeu-se que, em termos computacionais, essa representação utilizava melhor os recursos de processamento, visto que na execução do AG são criadas várias gerações. 
Com o ID do OA sendo representado por dez bits, é possível endereçar todo um repositório contendo até mil e vinte e quatro OAs $\left(2^{10}=1024\right)$. Isso permite que o repositório de OAs possa crescer sem causar problemas no AG. Essa captura automatizada é possível porque, cada vez que é inserido um novo OA através da interface do Moodle [22], é criado um diretório no sistema de arquivos para abrigar os arquivos do OA. O nome do diretório é exatamente o mesmo do campo id (chave primária) utilizado na tabela $m d l \_s c o r m$ do banco de dados do Moodle. Assim, é possível percorrer os arquivos de determinado OA no sistema de arquivos e, no momento da recomendação resultante do algoritmo genético, saber qual OA está sendo recomendado.

Outra forma de representar o ID do OA seria capturar a quantidade de OAs no repositório em tempo de execução e, a partir dessa informação, definir quantos bits seriam necessários para representação do OA. Porém, essa forma de representação mostrou-se com um custo de processamento muito elevado, pois, mesmo antes da execução do AG, seria necessário percorrer todos os arquivos do repositório apenas para definir o tamanho do ID do OA.

A aptidão de cada $\mathrm{OA}$, por sua vez, é representada por sete bits, podendo resultar em um valor de até cento e vinte e oito na representação decimal $\left(2^{7}=128\right)$. Quando da execução do AG, é considerada como aptidão do cromossomo a soma das aptidões dos três OAs do cromossomo. Ou seja, para efeito da escolha do indivíduo com maior possibilidade de reprodução (maior aptidão), é considerada a aptidão do cromossomo como um todo.

Essa abordagem de um cromossomo contendo três OAs foi utilizada porque, no final da execução do AG, será indicado um cromossomo como o indivíduo mais apto, ou seja, como a melhor solução levando-se em consideração o contexto em questão. Portanto, não seria interessante recomendar apenas um OA, mas sim um conjunto dentre o qual o estudante pudesse escolher aquele que lhe fosse mais conveniente. Dessa forma, o estudante tem a opção de escolher, dentre os OAs recomendados, aquele que lhe pareça realmente útil.

\subsubsection{Tamanho da população}

O tamanho da população utilizada depende da quantidade de OAs no repositório. Devido ao que já foi explicado anteriormente, a quantidade de cromossomos será correspondente à quantidade de OAs dividida por três.

Para a composição da população durante os testes, foram utilizados tanto OAs no padrão disponíveis em repositórios de OAs encontrados na Internet, como também OAs que possuíam apenas metadados. Nesse último caso, eram construídos manualmente apenas os metadados rela- tivos ao conteúdo fictício dos OAs, como se possuíssem o conteúdo propriamente dito. Essa foi uma alternativa para realização de testes com uma maior quantidade de OAs, e foi possível devido à utilização do padrão SCORM.

\subsubsection{Realização da mutação}

Quando ocorre uma mutação, troca-se uma característica do cromossomo em questão. No caso do AG implementado, uma característica representa o próprio $\mathrm{OA}$ em si. Podem ocorrer duas situações distintas quando da mutação: uma caso a quantidade de OAs no repositório possua uma divisão exata por três e outra caso a divisão não seja exata.

No caso da divisão ser exata, haverá um número $x$ de cromossomos e cada um deles conterá exatamente três OAs. Nesse caso, a mutação ocorre trocando-se, de forma aleatória, um OA em um cromossomo por outro OA que já pertença a outro cromossomo. Para tanto, deve-se sortear:

(i) a posição do cromossomo que sofrerá a mutação, ou seja, em que posição esta ocorrerá;

(ii) qual o cromossomo do qual será retirado o OA; e

(iii) qual a posição do OA neste mesmo cromossomo.

Por outro lado, caso não seja uma divisão exata, haverá uma quantidade $x$ de cromossomos completos, mais um ou dois OAs (não pode ser três, pois a divisão seria exata e entraria no caso citado no parágrafo anterior) em "reserva", ou seja, OAs que não pertencem a nenhum cromossomo. Nesse caso, quando da ocorrência da mutação, será escolhida uma posição aleatória do cromossomo que sofrerá a mutação e o OA pertencente a essa posição será trocado por um daqueles da reserva. Dessa forma, o OA que está em reserva terá a chance de participar do AG, podendo melhorar ou piorar a aptidão de determinado cromossomo.

\section{Considerações Finais e Trabalhos Futuros}

Um dos desafios do ensino não presencial é a adequação do conteúdo ao estudante de acordo com as características cognitivas deste. No ensino presencial, esta já é uma tarefa difícil de ser realizada, visto que é necessário que o professor possua certo conhecimento das necessidades de cada um dos estudantes. Tal tarefa torna-se ainda mais complexa quando o ensino é realizado a distância, visto que muitos ambientes virtuais de aprendizagem enfocam, geralmente, aspectos relacionados à sua funcionalidade, esquecendo assim a função pedagógica do ambiente.

Outro fator que deve ser levado em consideração é 
que, nem sempre, os recursos tecnológicos utilizados para o ensino permitem realizar o ensino de forma simples e completa, sendo necessárias algumas adaptações, como é o caso da aprendizagem móvel, a qual pode apresentar limitações de acordo com as características do dispositivo móvel do estudante.

Pensando nisso, o presente trabalho apresentou uma abordagem que, através da aplicação de um AG, realiza a recomendação de conteúdos educacionais (OAs) que sejam adequados às características do contexto do estudante. Para tanto, a abordagem leva em consideração uma série de características, conforme mostrado ao longo deste trabalho. O intuito desta abordagem é aperfeiçoar o processo de ensino-aprendizagem de forma transparente aos estudantes, provocando assim um maior interesse no estudo por parte destes.

Outra contribuição deste trabalho que merece destaque é o fato do ambiente ter sido desenvolvido utilizandose de um SMA que pode ser utilizado em outros contextos, ou seja, a arquitetura proposta pode ser utilizada em qualquer área de aplicação.

Por fim, a extensão adicionada ao MLE pode ser utilizada por outras pessoas que utilizem esse framework para desenvolvimento de suas aplicações, podendo assim utilizar a informação de localização geográfica do estudante como acharem necessário.

Apesar do trabalho realizado até o momento, há algumas melhorias que podem ser realizadas. Assim, como trabalho futuro, pretende-se:

- Testar o ambiente desenvolvido em um contexto real, utilizando os principais repositórios de conteúdos educacionais (OAs). Isso possibilitará mensurar, estatisticamente, o quanto este mecanismo de recomendação contribui para o processo de ensino-aprendizagem;

- Disponibilizar a infraestrutura do ambiente, denominado MobiLE, como um componente do Moodle sob uma licença GPL, de forma a facilitar a instalação e utilização do ambiente por instituições que utilizem esse LMS e desejem disponibilizá-lo através de dispositivos móveis;

- Testar o ambiente desenvolvido em áreas específicas (ex. área médica, petrolífera, etc.) para verificar a adequabilidade da recomendação de OAs em contextos específicos;

- Aperfeiçoar a visualização da recomendação, visto que no momento a recomendação é feita de forma textual, informando o nome do OA;
- Levar em consideração outras características do dispositivo móvel do estudante antes de decidir se um OA é adequado ao contexto do estudante, aperfeiçoando, consequentemente, o mecanismo de recomendação.

\section{Referências}

[1] Associação Brasileira de Educação a Distância ABED. CensoEAD.BR: Relatório Analítico da Aprendizagem a Distância no Brasil, São Paulo: Pearson, 2012.

[2] J. -K. Yau e M. Joy. A context-aware personalized m-learning application based on m-learning preferences. In Proceedings of 6th IEEE International Conference on Wireless, Mobile and Ubiquitous Technologies in Education (WMUTE), 11-18, 2010.

[3] P. Moore, B. Hu, M. Jackson e J. Wan. Intelligent context for personalized m-learning. In Proceedings of International Conference on Complex, Intelligent and Software Intensive System (CISIS), 247-154, 2009.

[4] E. R. Rodolpho. Convergência digital de objetos de aprendizagem SCORM. Dissertação de Mestrado, Universidade Estadual Paulista Júlio de Mesquita Filho (UNESP), São José do Rio Preto, SP.

[5] LTSC. Draft Standard for Learning Object Metadata. IEEE 1484.12.1-2002. 2002.

[6] R. Linden. Algoritmos Genéticos - Uma importante ferramenta da Inteligência Computacional, $2^{\text {a }}$ Edição. Brasport, Rio de Janeiro, 2008.

[7] R. Russell e P. Norvig. Artificial Intelligence, A Modern Approach 2nd Edition. Prentice Hall, 2003.

[8] A. A. A. Pontes. Uma Arquitetura de Agentes para Suporte à Colaboração na Aprendizagem Baseada em Problemas em Ambientes Virtuais de Aprendizagem. Dissertação de Mestrado, Universidade Federal Rural do Semiárido (UFERSA), Mossoró, RN, 2010.

[9] A. A. A. Pontes, F. M. Mendes Neto e G. A. L. de Campos. Multiagent System for Detecting Passive Students in Problem-Based Learning. Advances in Soft Computing, v. 71, 165-172, 2010.

[10] ADL. Advanced distributed learning. http://www.adlnet.org, Jun. 2011.

[11] C. C. L. Dias, A. Kemczinski e S. V. d. S. Lucena. Padrões abertos: aplicabilidade em Objetos de Aprendizagem (OAs). In Anais do XX Sim- 
pósio Brasileiro de Informática na Educação (SBIE), Florianópolis, SC, 2009.

[12] S. Petroli Neto. Computação Evolutiva: desvendando os algoritmos genéticos. Ubiquidade. Revista de Estudos de Tecnologia da Informação e Comunicações, 1(1):34-45, 2011.

[13] A. O. Artero. Inteligência Artificial - Teoria e Prática, $1^{\mathrm{a}}$. Edição. Livraria da Física, São Paulo, 2009.

[14] É. D. O. C. Zini. Algoritmo Genético Especializado na Resolução de Problemas com Variáveis Contínuas e Altamente Restritos. Dissertação de Mestrado, Universidade Estadual Paulista (UNESP), 2009.

[15] P. Moore, B. Hu e J. Wan. A Context Ontology for Pervasive Mobile Computing. The Computer Journal, 53, 191-207, 2008.

[16] B. Schilit, N. Adams e R. Want. Context-Aware Computing Applications. In Proceedings of IEEE Workshop on Mobile Computing Systems and Applications (WMCSA), Santa Cruz, 85-90, 1994.

[17] T. T. Primo, R. M. Vicari e J. M. C. Da Silva. Rumo ao Uso de Metadados Educacionais em Sistemas de Recomendação. In Anais do XXI Simpósio Brasileiro de Informática na Educação (SBIE), João Pessoa, PB, 2010.

[18] J.C. Gluz e R. M. Vicari. MILOS: Infraestrutura de Agentes para Suporte a Objetos de Aprendizagem OBAA. In Anais do XXI Simpósio Brasileiro de Informática na Educação (SBIE), João Pessoa, PB, 2010.

[19] JADE. Java development framework: an opensource platform for peer-to-peer agent based applications. http://jade.tilab.com/, Jun. 2011.

[20] MLE. Mobile Learning Engine. http://mle.sourceforge.net/mle/index.php, Ago, 2011.

[21] WURFL. Wireless Universal Resource FiLe”. http://wurfl.sourceforge.net/, Abril, 2011.

[22] MOODLE. About Moodle. http://docs.moodle.org/21/en/About_Moodle, Dez, 2011.

[23] A. Saccol, E. Schlemmer, J. Barbosa. m-learning e u-learning - Novas perspectivas da aprendizagem móvel e ubíqua. Pearson, São Paulo, 2010.

[24] M. Weiser. The computer for the 21 st century. Scientific America, 265(3):66-75, 1991.
[25] K. Mandula, S. R. Meda, R. Kambham. Implementation of Ubiquitous Learning System Using Sensor Technologies. In Proceedings of IEEE International Conference on Technology for Education, Hyderabad, India, IEEE Computer Society, pages 142-148, 2011.

[26] A. A. F. Loureiro et al. Computação Ubíqua Ciente de Contexto: Desafios e Tendências. In Anais do XXVII Simpósio Brasileiro de Redes de Computadores e Sistemas Distribuídos (SRBC'09), Recife, 2009.

[27] F. J. R.Vieira, M. A. S. N. Nunes. DICA: Sistema de Recomendação de Objetos de Aprendizagem Baseado em Conteúdo. Scientia Plena, 8, 2012.

[28] S. C. Cazella, K. K. Silva, P. Bhear, D. Schneider, R. Freitas. Recomendando Objetos De Aprendizagem Baseado em Competências em Ead. Novas Tecnologias na Educação, 9(2):110, 2011.

[29] H. N. J. Martins, E. B. Costa, T. T. M. Oliveira, I. I. Bittencourt. Sistema de Recomendação Híbrido para Bibliotecas Digitais que Suportam o Protocolo OAI-PMH. In Anais do XXII Simpósio Brasileiro de Informática na Educação, SBIE, Aracaju, 2011.

[30] I. Dickinson. Jena Ontology API. http://jena.sourceforge.net/ontology/index.html, Out. 2011.

[31] I. Bittencourt et al. Ontologia para Construção de Ambientes Interativos de Aprendizagem. In Anais do Simpósio Brasileiro de Informática na Educação (SBIE), Brasília, Páginas 567-576, 2006.

[32] W. X. Min, C. Wei, C. Lei. Research of Ontology-based Adaptive Learning System. In Proceedings of International Symposium on Computational Intelligence and Design (ISCID). Wuhan, China: IEEE Computer Society. Pages 366 -370, 2008.

[33] S. J. Rani, M. S. Ashok, K. Palanivel. Adaptive content for personalized E-learning using web service and semantic web. In Proceedings of International Conference on Intelligent Agent Multi-Agent Systems (IAMA). Pondicherry, India: IEEE Computer Society. Pages 1-4, 2009. 